\title{
Trust in the time of corona: epistemic practice beyond hard evidence
}

\author{
Jessica S. C. Leung ${ }^{1}$ (D) Maurice M. W. Cheng $^{2}$ (D)
}

Received: 31 August 2020 / Accepted: 17 March 2021 / Published online: 28 April 2021

(c) The Author(s), under exclusive licence to Springer Nature B.V. 2021

\begin{abstract}
The spread of the coronavirus disease 2019 (Covid-19) is undoubtedly one of the most extraordinary challenges in recent history. Amidst this global crisis, various controversies have been emerging about how to manage the virus, ranging from whether face masks should be required as a preventive measure to whether hydroxychloroquine is an appropriate treatment. There has been a barrage of contradictory claims related to these issues. However, in many cases, it is not possible for an individual to wait until consensus is reached before deciding on a course of action. Meanwhile, to avoid misplacing trust, trust must be well grounded. Conventional school science largely focuses on the trustworthiness of data and evidence, rather than that of the people making scientific claims. This failure to consider the human factor renders conventional school science inadequate for helping students make informed judgements about granting trust. Drawing on the literature in epistemic practice, this paper highlights four epistemic processes potentially useful for students to ground their trust, including (1) identifying whether recognition from peer reviewers has been obtained; (2) examining the credentials of those who claim expertise; (3) determining the level of expert consensus; and (4) identifying possible sources of bias. Through critical reflection on events related to the Covid-19 pandemic as examples, this paper examines how these epistemic processes inform judgement about the trustworthiness of people in terms of their competence and motives. The discussion highlights the need to develop students' capacity to identify expertise/ credentials, the nature of journals and of organisations when trust is assigned. This paper offers a frame for science educators on guiding students to place trust as a part of their decision-making process. The capability would be relevant to contexts beyond the Covid-19 pandemic.
\end{abstract}

Keywords Epistemic trust $\cdot$ Reliable epistemic processes $\cdot$ Socioscientific issues

Jessica S. C. Leung

leungscj@hku.hk

Maurice M. W. Cheng

maurice.cheng@waikato.ac.nz

1 The University of Hong Kong, Hong Kong, Hong Kong

2 Te Kura Toi Tangata School of Education, Divsion of Education, The University of Waikato, Hamilton, New Zealand 
The Covid-19 crisis has posed many questions and challenges. One of these has to do with decision-making regarding infection prevention behaviours (e.g. whether a person should dine out and if so, how often; whether a person should wear a face mask in public; whether a person should receive a Covid-19 vaccine). The crisis is unprecedented not only because the global scale of lockdowns has created social, economic and political unrest, but also because the rapid development of the science of the disease and the widespread use of social media to share information easily amplify any messages. It is not uncommon for individuals who use social media to receive an overwhelming amount of advice related to Covid-19 from friends and family. Recipients of such messages must then decide whether and the extent to which such advice is trustworthy.

The ability to evaluate the trustworthiness of advice pertaining to scientific or medical issues is an important capability that children start to develop at a young age. Educationespecially science education—should play a key role in this. Developing students' capability to evaluate scientific information serves not only to prepare students for the future, but also to guide them in their present-day practices when they are faced with the need to make informed decisions (Kuhn 2017).

Conventional school science teaches students to make evaluations about scientific information encountered in daily life, for example, the energy content of food (as indicated on food labels), the energy efficiency of electrical appliances (as indicated by relevant energy consumption information) and even which brand of chlorine bleach solution provides the most value (a concept that can be learned alongside lessons on titration). The curricular and pedagogical development pertaining to teaching socioscientific issues develops students' ability to make judgements and decisions in context (Sadler and Zeidler 2009), through activities such as searching for information, scientific inquiry and debate/argumentation. By learning the nature of science and scientific practice, students become better equipped to evaluate scientific claims (Leung 2020a).

Supporting students to make decisions and judgements based on evidence is not straightforward for two reasons. Firstly, it has been shown that when people make decisions on controversial issues that are inextricably tied with their worldviews or political views, even those who are highly numerate tended to interpret data in a way that would affirm or protect their existing views (Kahan et al. 2017). This phenomenon of motivated reasoning was also observed in educational contexts (e.g. Henderson and Duggan-Haas, 2014). Secondly, science and technology nowadays are so complex that even scientists find it challenging to evaluate the validity of scientific claims beyond their immediate field of expertise. It is thus a tall order to expect students to be able to evaluate the validity of science-related information they are exposed to in the mass media. This paper responds to Fensham's (2014) call for more work on teaching students 'when and how to put trust in science' (p. 656) and 'when to be sceptical about science' (p. 659). We attempt to address his call by discussing the notion of epistemic trust in the context of Covid-19. We use the concept of reliable epistemic processes to tackle the issue of misplacing trust in science and discuss what epistemic trust can offer to science education as practice.

\section{Epistemic trust}

In traditional school science and practical work settings, establishing the trustworthiness of claims focuses on evaluating experimental findings. For example, we trust that a solution is acidic because it turns a $\mathrm{pH}$ indicator red, and we trust that copper is a better electrical 
conductor than iron because, assuming the same length and thickness, the resistance of copper is lower.

Relying on empirical evidence is a key scientific practice. However, it is limited as a basis for evaluation when students are confronted with scientific claims for which the evidence is too complicated and the theoretical underpinnings too sophisticated for people outside the frontier research area to grasp. In such cases, students need to make decisions involving issues for which they are unable to examine conceptual coherence, the validity of the claims or the process behind the formulation of such claims. Inevitably, this requires them to place their trust based on aspects beyond 'hard evidence'.

The traditional science perspective does not fully acknowledge the human factor involved in trustworthiness, so from this viewpoint, trust is decontextualised. However, both psychological and philosophical perspectives acknowledge that trust is a relational concept. Trust emerges between a trusting person A and a trustee B who is entrusted by A with the care of something (C) for which A cannot care sufficiently himself/herself (Baier 1986, p. 237). This paper specifically focuses on epistemic trust, i.e. trust in relation to knowledge claims. In the context of this paper, 'persons A' are lay citizens, 'trustees B' are those who make knowledge claims (in particular, scientists and organisations) and 'something (C)' is the range of knowledge claims made in relation to Covid-19. A key element of assigning trust is the prior consideration not only of the validity of knowledge claims (which, in fact, people sometimes lack the ability to consider), but also of factors related to the trustees.

Stephen Norris (1995) argued that 'students need to be taught first that the object of their scepticism should be the believability of experts, not the evidence supporting scientific knowledge claims' (p. 216). This notion is echoed by Allchin (2020) who argued that placing trust in experts entails assessing people. We suggest that it is important to consider the competence and motives of those who claim expertise (Jones 1996). Prior studies have suggested that when educated laypersons are equipped with the ability to consider the factors of peer review, professional recognition, expert consensus and conflict of interest, they are more capable of evaluating scientific claims made in news reports (Leung 2020b). These factors embody the competence and motives of a trustee (see Table 1). The competence of experts is manifested in the recognition obtained from peer reviewers (i.e. peer review), the level and relevance of expertise (i.e. professional recognition) and the level of agreement by third-party experts (i.e. expert consensus). The motives of experts can be reflected in the presence or absence of vested interests (i.e. conflict of interest). By reflecting on the Covid-19 pandemic, we explore how these factors (or epistemic processes) work to support the examination of experts' competence and motives. This in turn may help outsiders to science judge which claims to trust and thus make more informed decisions.

Case 1: The association between 5G technology and Covid-19

The high infectivity of Covid-19, coupled with the widespread use of mainstream and social media, has created an environment that is unprecedentedly favourable for pseudoscience and conspiracy theories to proliferate. One claim that circulated recently was that Covid-19 is caused by $5 \mathrm{G}$ telecommunications networks. Claims about the health implications of telecommunications networks are not new: doctors experienced 'radiophobia' as early as 1903, and there were widespread fears in the 1990s that radiation from $2 \mathrm{G}$ telecommunications technology could cause cancer. During the Covid-19 crisis, the claims were made that radiation from $5 \mathrm{G}$ technology weakened people's immune systems, making them more vulnerable to Covid-19, and that $5 \mathrm{G}$ radiation directly transmitted the SARS$\mathrm{CoV} 2$. Amidst such rumours, protesters in Europe vandalised 5G cell towers. 


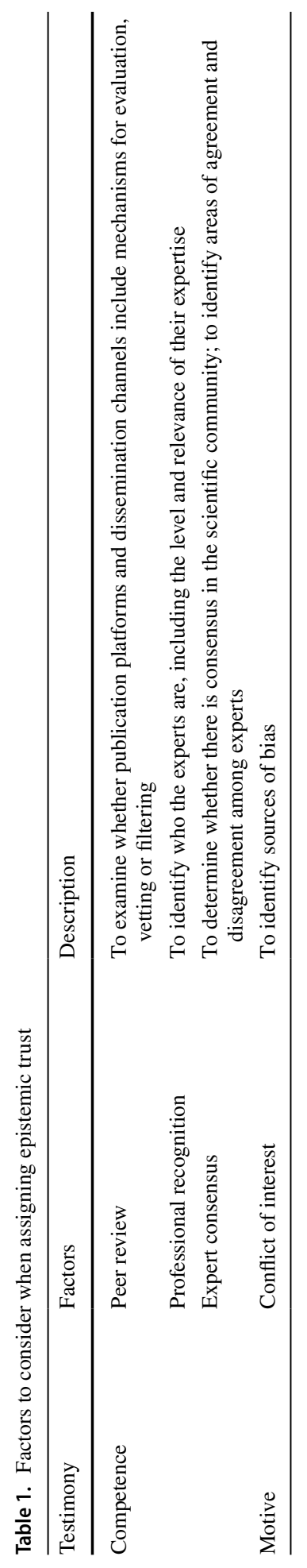


The drawing of an association between 5G technology and Covid-19 also appeared in an academic journal, making the question of whether to place trust in such claims even trickier. PubMed is a free life science and biomedical database that is accessible by both the general public and medical professionals. It is generally regarded as a source of reliable and genuine medical information rather than pseudoscientific scams. A paper titled '5G Technology and Induction of Coronavirus in Skin Cells' was published in the Journal of Biological Regulators and Homeostatic Agents that was indexed in PubMed. The affiliations of the authors did not raise any concerns, and the authors were from Guglielmo Marconi University, Central Michigan University and First Moscow State Medical University. Nevertheless, the journal was a predatory journal, i.e. a journal that did not properly subject submitted articles to peer review, but rather charged authors publication fees. Moreover, on closer examination, there were a number of causes for concern. None of the authors had the relevant expertise to make claims about the causes of Covid-19. The journal did not list the affiliations of its editorial board nor their full names, instead including only each member's initials, surname and country. Finally, J. W. Miers, one of the assistant editors, had been previously implicated in research misconduct (Panka et al. 2019). The inclusion of this predatory journal in PubMed suggested serious flaws in the approval process used for the index. PubMed eventually removed the article from its database, although it did not remove other publications from the same journal.

Cargo cult science is a term coined by Richard Feynman (1974) in his commencement speech at the California Institute of Technology. It more or less summarises the nature of that paper:

In the South Seas, there is a cargo cult of people. During the war, they saw airplanes land with lots of good materials, and they want the same thing to happen now. So they have arranged to imitate things like runways, to put fires along the sides of the runways, to make a wooden hut for a man to sit in, with two wooden pieces on his head like headphones and bars of bamboo sticking out like antennas- he is the controller - and they wait for the airplanes to land. They are doing everything right. The form is perfect. It looks exactly the way it looked before. But it does not work. No airplanes land. So I call these things cargo cult science, because they follow all the apparent precepts and forms of scientific investigation, but they are missing something essential, because the planes do not land.

Similar to the society described by Feynman that made bamboo radio antennas and imitation landing strips in the hopes of reattaining an influx of cargo goods, the findings that linked 5G to Covid-19 looked like science, but lacked scientific integrity and replicability. We believe that when outsiders to science assess the degree of trust to place in a claim, it is important that they are able to examine the credentials of parties associated with the claim (i.e. professional recognition). In this case, these parties included the authors, who could have been evaluated based on whether they had the expertise to speak about the causes of Covid-19. It also included the members of the journal's editorial board, whose track records and institutional affiliations could have been looked into. Merely examining whether the journal included a vetting mechanism (i.e. peer review) did not seem to be adequate as the predatory journal had a peer review system in place despite its questionable quality. It would be useful for a person faced with such a claim to have the ability to distinguish predatory journals and identify the credentials and affiliations of a journal's editorial board members.

Case 2: A crisis of trust-the World Health Organisation (WHO) 
While the first case is situated in pseudoscience and cargo cult science, Case 2 is embedded in authentic science. We refer to the controversy about the use of face masks and the role of WHO. Up to March 2020, WHO did not advise healthy people to wear masks unless they were infected or took care of infected people. It was only in June 2020 that WHO finally advised governments to encourage the public to wear fabric masks in situations where social distancing was difficult; people over the age of 60 or with underlying health risks were advised to wear medical masks (WHO 2020). Since the beginning of the outbreak, the use of masks or face coverings among individuals not medically diagnosed with Covid-19 has been a controversial issue around the world. Many countries such as Australia, Canada and the USA have recommended against the use of face masks for healthy individuals. In contrast, many places in Asia such as China, Indonesia, and the Philippines, Hong Kong and Taiwan have recommended its widespread use as a precaution to limit the spread of Covid-19. In Hong Kong, as early as mid-January 2020, the government advised people visiting hospitals for any reason to wear surgical masks and observe strict personal and hand hygiene (Hong Kong SAR Government 2020). People who support the use of face masks believe they give a modest but valuable level of protection, whereas opponents believe that such protection offers a false sense of security and makes users less vigilant about proven measures such as social distancing and handwashing. These opposing opinions have led to confusion over the use of face masks to limit the spread of Covid-19.

This case demonstrates that the public needs help making decisions amidst uncertainties and lack of evidence. Although evidence-based recommendations are ideal, it is not always possible for individuals to wait until the presence of uncontroversial evidence before one makes a decision about science-related advice, such as whether or not to wear a face mask. In such cases, following the precautionary principle is more beneficial than waiting for conclusive evidence. In the case of wearing face masks during the Covid-19 crisis, the potential benefits of wearing them outweigh the potential risks and it is thus better to err on the side of caution. As suggested by WHO, 'the precautionary principle states that in the case of serious or irreversible threats to the health of humans or the ecosystem, acknowledged scientific uncertainty should not be used as a reason to postpone preventive measures' (Martuzzi and Tickner 2004, p. 1). When applying the precautionary principle, one should bear in mind that advice given based on this approach should not be deemed final or irreversible, but should rather be considered temporary guidelines issued while awaiting the availability of more conclusive evidence. The precautionary principle was not reflected in the WHO recommendation made in March 2020.

Given that WHO comprises medical doctors, public health specialists, scientists and epidemiologists, it is reasonable to assume its competence. It would be useful to consider the nature and role of WHO and its track record in dealing with pandemics. WHO is an intergovernmental organisation that is part of the United Nations. Its mission is to "promote health, keep the world safe and serve the vulnerable' (WHO, no date). As a multilateral organisation, it has more than 194 member states (WHO 2019), with the US government and the Bill and Melinda Gates Foundation as its two biggest financial contributors (Cueto, Brown and Fee 2019, pp. 326-327). Unlike the World Trade Organisation and the North Atlantic Treaty Organisation, WHO does not have direct authority to regulate its member countries. Inevitably, its actions and decisions are likely to be informed by a variety of factors beyond the realm of science. There is consensus among historians and public opinion that its leadership was instrumental in eradicating smallpox in the late 1970s and in managing the SARS outbreak in 2002-2003. However, its management of the H1N1 pandemic in 2009 and the Ebola outbreak from 2014 was highly controversial (Cueto et al. 2019; Buranyi 2020). We therefore cannot assume that WHO is an authoritative organisation 
that is always competent in handling health crises. This points to the issue of professional recognition.

Given the severe global shortage of face masks at the beginning of the outbreak, it is possible that, had WHO followed the precautionary principle and recommended all people to wear face masks, fear would have spread among those who did not have access to them. Such advice would also have additionally stretched the limited supply of medical masks for frontline health workers. However, it is debatable whether it was morally sound of the organisation to disregard the precautionary principle and recommend that healthy people not wear face masks despite the lack of supporting evidence for this position. By identifying the possible sources of bias (i.e. conflicts of interest) that might have caused WHO to deviate from the precautionary principle, individuals would have been in a better position to judge WHO's trustworthiness.

An additional instance to consider is the comment by Dr Maria Van Kerkhove, head of the WHO's emerging diseases and zoonosis unit, on June 8, 2020 that asymptomatic transmission is 'very rare'. In WHO vaccine manual (2013), the use of the phrase 'very rare' refers to prevalence in less than $0.01 \%$ of a given population. Contrary to this comment, several studies released prior to the moment when the comment was made have proven that asymptomatic spread does happen. For instance, a study published in the Annals of Internal Medicine on June 3, 2020, estimated that about $40 \%$ to $45 \%$ of people infected with Covid19 will remain asymptomatic and will unknowingly spread the virus (Oran and Topol 2020). When Kerkhove made that comment, many health professionals raised concerns about it, including Dr Anthony Fauci, director of the National Institute of Allergy and Infectious Diseases in the USA, who stated that describing asymptomatic transmission as 'very rare' was 'not correct'. Kerkhove later expressed regret over her earlier comment and clarified that asymptomatic carriers contribute to the spread of the virus, but stated that more information was required to know how much they contributed. Despite this, her original comment detracted from WHO's perceived competence as reflected in the contrasting claims made in published findings (i.e. peer review) and by third-party experts (i.e. expert consensus).

Case 3: Blunders in science-the hydroxychloroquine treatment

Finally, let us consider two retracted articles on medication related to Covid-19 from two leading journals. The New England Journal of Medicine (NEJM) article 'Cardiovascular Disease, Drug Therapy, and Mortality in Covid-19' examined the effect of certain heart medications on Covid-19 patients and found no safety concerns. The Lancet article, titled 'Hydroxychloroquine or Chloroquine with or without a Macrolide for Treatment of Covid19: A Multinational Registry Analysis', claimed that hydroxychloroquine, an antimalarial drug, increased the risk of death when used to treat Covid-19. Both papers were from the same group of authors relying on existing data gathered from hundreds of hospitals around the world. These data had been gathered by Surgisphere, a company founded by one of the authors, an interest that was declared in each paper. Some scientists were highly sceptical of the Lancet paper, especially with regard to the source of its data set and how it had been analysed. In an open letter, more than 145 scientists stated that it was unlikely that the company had obtained the hospital records alleged to be in the database given that no one else had access to this information. In response, the other three authors requested for an independent third-party review of the data from Surgisphere to replicate the analyses made in the article. However, the company refused to make its raw data available to thirdparty reviewers for validation out of concern that such data transfer would violate client agreements and confidentiality requirements. Without access to the raw data, third-party reviewers were not able to conduct the review. Hence, the authors requested both articles to 
be retracted. By early June, both the Lancet and NEJM articles were retracted. Ironically, the day after the retraction, the basic conclusion of the Lancet study was supported by a randomised controlled trial involving more than 4,600 patients.

By and large, having obtained recognition from peer reviewers, findings in academic journals are expected to be reasonably trustworthy. However, the peer review system is imperfect despite its merits. Fortunately, as soon as findings are published, they are open to scrutiny by the science community. Judging the level of consensus reached in the science community enables outsiders to science to assign their trust accordingly. Admittedly, it takes time for the science community to raise concerns, and it is possible for trustees to fail expectations even when they are competent (as reflected in this case by the recognition obtained by the authors from peer reviewers in leading journals) and driven by good motives (as reflected in this case by their disclosure of vested interests and their initiative to request for a third-party review in response to the open letter). Yet it is appreciable that it took only 13 days from the time the paper was published to the time it was retracted in response to the concerns raised by scientists through the open letter.

This case demonstrates that there is no magic bullet for deciding when to grant trust. Prior to the publication of the open letter, lay members of the public strictly following reliable epistemic processes would not have been able to know that the trustee had failed their trust. It is only because of the subsequent events concluding with the retraction of the peerreviewed paper that we have retrospectively been able to identify that the consideration of expert consensus was relevant for judgements about reassigning trust in that case. Notably, although reliable epistemic processes can help people make informed decisions about trustworthiness at the moment they encounter a knowledge claim, such decisions may not necessarily be the 'right' ones.

\section{What have we learned about placing trust in the time of corona?}

In the highly specialised and large-scale epistemic endeavour centred around Covid-19, the main issue surrounding trust is not whether we should trust others, but who to place trust and how much trust should be granted. By reflecting on the Covid-19 pandemic, this paper examined how reliable epistemic processes work to help a layperson evaluate the competence and motives of those who claim expertise, and therefore whether and to what degree their specific claims can be considered trustworthy. Competence can be assessed by the following: (1) identifying the credentials of experts (e.g. the level and relevance of expertise) as in the case of the authors of the retracted paper that associated 5G technology with Covid-19 (i.e. professional recognition); (2) determining whether there is consensus in the science community, as in the case of the scepticism about the data set from Surgisphere and the disagreement surrounding WHO's comment that asymptomatic transmission is 'very rare' (i.e. expert consensus); and (3) examining whether publication platforms and dissemination channels include mechanisms for evaluation, vetting or filtering (i.e. peer review). These epistemic processes are intrinsic to the social context in which science knowledge is generated and have previously been found to be within students' abilities (Leung et al. 2017), despite their low adherence to these processes in their evaluation of scientific claims (Leung 2020c).

This paper adds to the literature of developing students' epistemic practice in science in two ways. First, it highlights the need to transcend a mere examination of whether findings have undergone a peer review process. As suggested in the case of cargo cult science that 
associated 5G technology with Covid-19, helping students identify predatory journals is one way to prevent them from blindly trusting peer-reviewed findings. Second, it emphasises the importance of evaluating motives that may be manifested in the possible sources of bias involved in shaping a claim (i.e. conflict of interest), as in the case of WHO's hesitation to recommend the use of face masks. Our discussion demonstrates that understanding the nature of an organisation (e.g. WHO's orientation and its stakeholders) can help outsiders identify possible biases embedded in a claim. We concur with Tom Bryce and Stephen Day (2014) that 'science teachers need to support students' learning towards being able to identify and reason through potentially biased information, whether it is political, ideological or, in the case of some scientists, confirmation bias' (p. 1032).

Each of the three cases requires different sets of reliable epistemic processes to help a person make decisions about assigning trust, demonstrating that some reliable epistemic processes are more useful than others in specific contexts. Through critical reflection on these three cases in the context of the Covid-19 pandemic, this paper illustrated how reliable epistemic processes may equip students to make informed decisions about trusting people who offer knowledge claims. It challenged the way conventional school science has largely focused on the trustworthiness of data and evidence rather than also considering the trustworthiness of people in science. It demonstrated that the consideration of this human factor makes it possible to make an informed judgement about placing trust. In light of the current global crisis, reliable epistemic processes-peer review, professional recognition, expert consensus and conflict of interest-may help students assess the competence and motives of those making knowledge claims, thus enabling them to make informed decisions about placing trust amidst uncertainties and controversies. We are aware that worldviews or political stances contribute to reasoning when people determine competences and motives of 'experts'. Existing studies were contextualised in climate change, pollution, energy issues, etc., in which the contexts do not have immediate life and death implications. While Covid-19 is still a big threat to us, it creates an opportunity for us to examine the way that people place trust and make decisions when they are confronted with immediately life-and-death issues. We do not think Covid-19 is the last pandemic or global health challenge that we human beings face. It would be important for us to better understand the way that we place trust, and to better equip students to live up to these challenges.

\section{References}

Allchin, D. (2020). The covid-19 conundrum. The American Biology Teacher, 82(6), 429-433. https://doi. org/10.1525/abt.2020.82.6.429.

Baier, A. (1986). Trust and antitrust. Ethics, 96(2), 231-260. https://doi.org/10.2307/2381376.

Bryce, T. G., \& Day, S. P. (2014). Scepticism and the science of global warming: A rejoinder. Cultural Studies of Science Education, 9(4), 1025-1037. https://doi.org/10.1007/s11422-014-9651-7.

Buranyi, S. (2020, 10 April). The WHO v coronavirus: Why it can't handle the pandemic. The Guardian. Retrieved from: https://www.theguardian.com/news/2020/apr/10/world-health-organization-who-vcoronavirus-why-it-cant-handle-pandemic

Cueto, M., Brown, T. M., \& Fee, E. (2019). The world health organization: A history. Cambridge, England: Cambridge University Press.

Fensham, P. J. (2014). Scepticism and trust: Two counterpoint essentials in science education for complex socio-scientific issues. Cultural Studies of Science Education, 9(3), 649-661. https://doi.org/10.1007/ s11422-013-9560-1.

Feynman, R. P. (1974). Cargo cult science. Engineering and Science, 37(7), 10-13. 
Hong Kong SAR Government. (2020, 16 January). CHP closely monitors confirmed case of infection of novel coronavirus in Japan and revises reporting criteria. Retrieved from: https://www.info.gov.hk/ gia/general/202001/16/P2020011600659.htm

Henderson, J. A., \& Duggan-Haas, D. (2014). Drilling into controversy: The educational complexity of shale gas development. Journal of Environmental Studies and Sciences, 4(1), 87-96. https://doi.org/10. 1007/s13412-013-0161-9.

Jones, K. (1996). Trust as an affective attitude. Ethics, 107(1), 4-25. https://doi.org/10.1086/233694.

Kahan, D. M., Peters, E., Dawson, E., \& Slovic, P. (2017). Motivated numeracy and enlightened self-government. Behavioural Public Policy, 1(1), 54-86. https://doi.org/10.1017/bpp.2016.2.

Kuhn, D. (2017, 21 December). What makes a student successful? [video file] Retrieved from: https://globa lcenters.columbia.edu/news/deanna-kuhn-lecture-and-new-book-signing-event-held

Leung, J. S. C. (2020a). A practice-based approach to learning nature of science through socioscientific issues. Research in Science Education. https://doi.org/10.1007/s11165-020-09942-w.

Leung, J. S. C. (2020b). Promoting students' use of epistemic understanding in the evaluation of socioscientific issues through a practice-based approach. Instructional Science, 48, 591-622. https://doi.org/10. 1007/s11251-020-09522-5.

Leung, J. S. C. (2020c). Students' adherences to epistemic understanding in evaluating scientific claims. Science Education, 104(2), 164-192. https://doi.org/10.1002/sce.21563.

Leung, J. S. C., Wong, A. S. L., \& Yung, B. H. W. (2017). Evaluation of science in the media by nonscience majors. International Journal of Science Education, Part B, 7(3), 219-236. https://doi.org/10. 1080/21548455.2016.1206983.

Martuzzi, M., \& Tickner, J. A. (Eds.). (2004). The precautionary principle: Protecting public health, the environment and the future of our children. Copenhagen, Denmark: World Health Organization.

Norris, S. P. (1995). Learning to live with scientific expertise: Towards a theory of intellectual communalism for guiding science teaching. Science Education, 79, 201-217. https://doi.org/10.1002/sce.37307 90206.

Oran, D. P., \& Topol, E. J. (2020). Prevalence of asymptomatic SARS-CoV-2 infection: A narrative review. Annals of Internal Medicine. https://doi.org/10.7326/M20-3012.

Panka, D. J., Wang, W., Atkins, M. B., \& Mier, J. W. (2019). Retraction: The raf inhibitor BAY 43-9006 (Sorafenib) induces Caspase-independent apoptosis in melanoma cells. Cancer Research, 79(20), 5459. https://doi.org/10.1158/0008-5472.CAN-19-2621.

Sadler, T., \& Zeidler, D. L. (2009). Scientific literacy, PISA, and socioscientific discourse: Assessment for progressive aims of science education. Journal of Research in Science Teaching, 46(8), 909921. https://doi.org/10.1002/tea.20327.

WHO. (no date). Our values. Retrieved from: https://www.who.int/about/who-we-are/our-values

WHO. (2019). Countries. Retrieved from: https://www.who.int/countries/en/

WHO. (2020). Advice on the use of masks in the context of COVID-19. Retrieved from: https://apps.who. int/iris/rest/bitstreams/1279750/retrieve

World Health Organization. (2013). Vaccine safety basics learning manual. Geneva, Switzerland: WHO.

Publisher's Note Springer Nature remains neutral with regard to jurisdictional claims in published maps and institutional affiliations.

Jessica S. C. Leung is an Assistant Professor in the Faculty of Education, The University of Hong Kong. Her current research interests are focused on epistemic practice and socioscientific issues, in particular how students can be better supported to make informed judgements on controversial issues, and the learning and teaching of nature of science.

Maurice M. W. Cheng is an Associate Professor in the Division of Education, The University of Waikato. He is interested in science and chemistry education, in particular, student learning of socioscientific issues. His research interests include the role of visual representations in learning and how drawing serves as a tool for learning and in research. 